978-1-7281-3199-3/20/\$31.00 (C)2020 IEEE

M. Vandemaele, K.-H. Chuang, E. Bury, S. Tyaginov, G. Groeseneken and B. Kaczer, "The Influence of Gate Bias on the Anneal of Hot-Carrier Degradation," 2020 IEEE International Reliability Physics Symposium (IRPS), 2020, pp. 1-7, doi: 10.1109/IRPS45951.2020.9128218. (https://ieeexplore.ieee.org/document/9128218) 


\title{
The Influence of Gate Bias on the Anneal of Hot-Carrier Degradation
}

\author{
Michiel Vandemaele ${ }^{* \dagger}$, Kai-Hsin Chuang ${ }^{* \dagger}$, Erik Bury ${ }^{\dagger}$, Stanislav Tyaginov ${ }^{\dagger \ddagger}$, Guido Groeseneken*†, Ben Kaczer ${ }^{\dagger}$ \\ *ESAT, KU Leuven, Leuven, Belgium \\ Email: michiel.vandemaele@imec.be \\ †imec, Leuven, Belgium \\ $\ddagger$ Institute for Microelectronics, TU Wien, Vienna, Austria \\ $\S$ Ioffe Physical-Technical Institute, St.-Petersburg, Russia
}

\begin{abstract}
We observe that non-zero gate bias applied during a high temperature anneal following hot-carrier degradation (HCD) impacts degradation recovery in nFETs. The devices are arranged into custom-built arrays and fabricated in a commercial $40 \mathrm{~nm}$ bulk CMOS technology and the FET anneal is induced by on-chip poly-Si heaters. The anneal is modeled using Stesmans' passivation model for $P_{\mathrm{b}}$-defects in hydrogen gas $\left(\mathrm{H}_{2}\right)$. Negative gate bias improves the anneal, in line with studies on biased passivation of process-induced $P_{\mathrm{b}}$-defects.
\end{abstract}

Index Terms-Hot-carrier degradation, anneal, recovery, $P_{\mathrm{b}}$ centers

\section{INTRODUCTION}

Hot-carrier degradation (HCD) is a metal-oxidesemiconductor field-effect transistor (MOSFET) ageing mechanism occurring when the transistor is biased in the on-state at high drain voltage. Under this condition, carriers at the drain side are accelerated to high energies. Upon collision with the oxide-semiconductor interface, they can break $\mathrm{Si}-\mathrm{H}$ bonds and form defects ( $P_{\mathrm{b}}$-centers). The charged defects will distort the $I-V$ characteristics of the device (a change in the threshold voltage, on-current, subthreshold slope). Due to continued device scaling at approximately constant supply voltage, HCD is becoming critical in recent technology nodes [1], [2].

Although typically assumed more permanent than bias temperature instability (BTI), HCD can be (partially) recovered by exposing the devices to an anneal at increased temperatures. HCD anneal has been widely studied in the past, using a variety of heat sources. A first possibility is to use a furnace or a heated chuck to reach the anneal temperature [3]-[7]. Secondly, an external microheater can be stacked [8] or monolithically integrated [9] on a chip to induce HCD anneal. A third option is to exploit the self-heat caused by currents of the device-under-test (DUT). Self-heat originating from bodydrain current [10], punch-through current [11] and current through (the length of) the gate (using dual gate pads) [12] are suitable for HCD anneal. Finally, poly-Si heaters surrounding the DUT have also been used, but for long gate lengths $\left(L_{\mathrm{g}}\right.$ $=6 \mu \mathrm{m})[13]$.
To fully benefit from HCD anneal, it is important to understand which factors influence (improve) the anneal. Using the model of Stesmans [14], Pobegen et al. [13] and de Jong et al. [6] connected recovery of HCD during anneal to a repassivation of the $P_{\mathrm{b}}$-centers created during hot-carrier (HC) stress. Investigations of process-induced $P_{\mathrm{b}}$-centers by Ragnarsson and Lundgren [15] showed that the gate bias applied during post metallization anneal influences the passivation. This rises the question whether an applied gate bias influences HCD anneal as well. In the literature, results on biased (i.e. non-zero gate voltage) HCD anneal are sparse. Young [16] reported biased HCD anneal with positive gate voltage only for long channel $\left(L_{\mathrm{g}}=6 \mu \mathrm{m}\right)$ poly-Si thin-film-transistors and MOSFETs. Parthasarathy et al. [17] studied the effect on pFETs of nBTI applied after HC stress and thus the evolution of HCD at fixed (high negative) gate bias when the drain bias is removed.

The purpose of this work is to investigate how gate bias impacts HCD anneal. To this end, we perform stress and anneal (heat source $=$ poly-Si heaters) measurements on a chip in a commercial $40 \mathrm{~nm}$ bulk CMOS technology. We $i$ ) develop a strategy for measuring HCD anneal on the chip, ii) confirm the anneal on chip and that it can be described using Stesmans' model and iii) find the anneal to strongly depend on gate bias. The results are of interest for augmenting the HCD Si-H bond breakage model [18], [19], self-healing electronics [20] and age monitoring circuits [21].

\section{Methodology}

First, we present the measured chip. Then, we discuss how the series resistance and pass gates on the chip can complicate the measurement. Finally, we give an overview of the measurement procedure.

\section{A. Chip}

We use a chip in commercial bulk $40 \mathrm{~nm}$ CMOS technology originally designed for physically-unclonable function (PUF) studies [22] (Fig. 1). The chip contains 4 arrays of $32 \times 16$ (rows $\times$ columns) planar nFETs and each array has devices with a different gate length $\left(L_{\mathrm{g}}=40,100,200\right.$ and $400 \mathrm{~nm}$, 


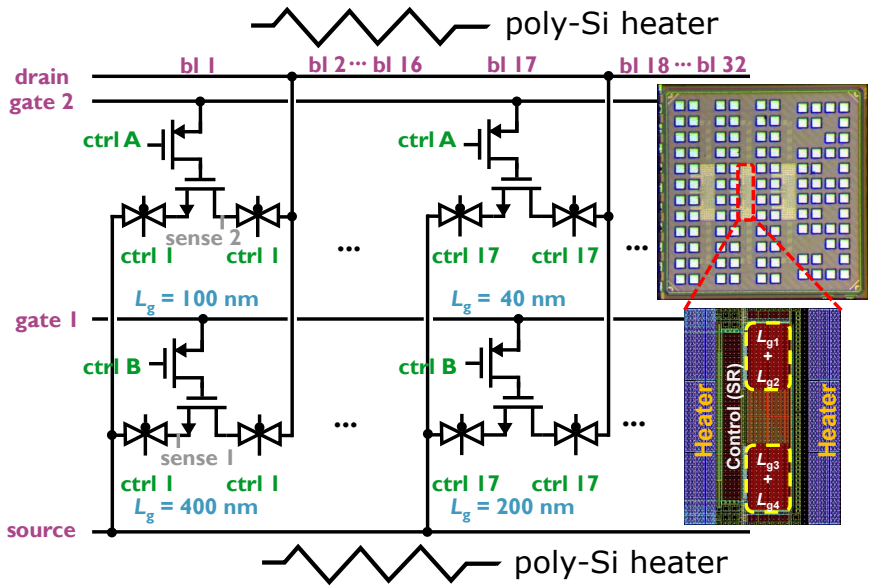

Fig. 1. Circuit schematics of the chip. Each device-under-test (nFET) is a parallel combination of 32 minimum width devices $(W=120 \mathrm{~nm})$ and has a pFET selector connected to its gate. Each column (bit line $=\mathrm{bl}$ ) is repeated 16 times, but the two sense lines are only present in the first column. Right: Micrograph (top) and layout (bottom) of the chip.

$W=120 \mathrm{~nm}$ ). A pFET selector allows to connect the gate of an nFET DUT to the gate line or leave it floating. All columns in an array have a full pass gate $(\mathrm{nFET}+\mathrm{pFET})$ on the source and drain lines. The chip contains poly-Si heaters, which allow very fast $(\Delta t \sim 10 \mathrm{~ms}$ ) heating to temperatures $\Delta T \sim 300 \mathrm{~K}$ above the chuck temperature [23]. The temperature increase of the DUT is calculated from the heater power based on a calibration with a dedicated on-chip diode [22].

We are limited to measuring the 32 FETs in one column in parallel as one large DUT (Fig. 1). Measuring each FET individually is infeasible due to offset current caused by the unselected FETs, of which the gate voltage is floating and cannot be set to zero.

\section{B. Series resistance and pass gates}

We now quantify the series resistance of the DUTs on the chip. The chip contains two sense lines (labeled 'sense 1' and 'sense 2' in Fig. 1), which allow measuring the actual voltage arriving on the source or drain of specific DUTs. By measuring the sense lines during an $I-V$-sweep of these DUTs, the voltage drop over the total series resistance and the value of the total series resistance can be calculated (Fig. 2b). This series resistance is estimated to be $90 \Omega$ and consists of two parts: one originating from the pass gate transistors and one caused by other sources. The pass gate component depends on the supply voltage $V_{\mathrm{dd}}$ of the chip. We extract its value by performing circuit simulations of the combination of pass gates and the DUT in Spectre ${ }^{\circledR}$ [24] using the foundry process design kit (PDK). For a $V_{\mathrm{dd}}=1.5 \mathrm{~V}$, the pass gate contribution is $20 \Omega$, making the additional series resistance $70 \Omega$.

Since we measure large DUTs $(1$ column $=32$ minimum width devices), of which the size is comparable to the size of the pass gate transistors, there is a concern that the pass gates cannot supply sufficient current. Normally, pass gates are much wider than the actual DUT and therefore influence
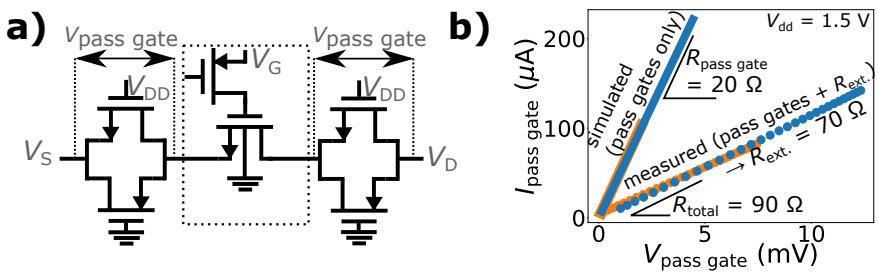

Fig. 2. a) Schematic of the DUT (with pFET selector, dashed box) and its pass gates on the source and drain. b) Simulated and measured (using the sense lines during a linear $I-V$ of the DUT) $I-V$ of the source and drain pass gate. The difference between the measured $R_{\text {series }} \sim 90 \Omega$ and the simulated $R_{\text {series }} \sim 20 \Omega$ allows to determine the additional series resistance $(\sim 70 \Omega)$ besides the pass gate resistance.

the current of the DUT very little (ideally not at all) and just connect or disconnect the DUT to/from the rest of the circuit.

To assess the influence of the pass gates on our large DUTs, we performed circuit simulations in Spectre ${ }^{\circledR}$ [24] of the oncurrent of the DUT without and with the pass gates (Fig. 3a). As can be seen, the pass gates decrease the on-current of the DUT. Fig. $3 b$ shows the relative decrease for our wide DUTs and for hypothetical DUTs of smaller width. We see that the influence of the pass gates on the DUT's on-current would go away if we could measure DUTs of a smaller width. The influence of the pass gates is less pronounced when sensing at the threshold voltage compared to in the on-state (since the current level is lower). Increasing the supply voltage $V_{\mathrm{dd}}$ of the chip can also improve the situation.

We also need to consider that the pass gates, and thus the series resistance of the DUTs, can degrade during the anneal. Indeed, the pass gates experience electrical stress at high temperature $\left(T_{\max }=270{ }^{\circ} \mathrm{C}\right)$ during the anneal. To assess the influence of this degradation, we carried out circuit simulations in Spectre ${ }^{\circledR}$ [24] of the relative change in DUT current for a certain degradation of the pass gates (Fig. 4). The pass gate degradation was modeled as an equal threshold voltage shift $\Delta V_{\text {th,pass gate }}$ applied to all its four transistors (nFET and pFET at both the source and drain side). This was achieved in the circuit simulations by correcting the gate voltage of the pass gate nFETs to $V_{\mathrm{dd}}-\Delta V_{\mathrm{th}}$,pass gate and the gate voltage of the pass gate pFETs to $0 \mathrm{~V}+\Delta V_{\text {th,pass gate }}$ (see Fig. 2a). The circuit simulation was done for different values of $\Delta V_{\mathrm{th} \text {,pass gate }}$, corresponding to different degrees of pass gate degradation. The results (Fig. 4b) show that, independent of the exact value of the pass gate degradation, the change in the DUT current is less severe for a sense voltage at the threshold voltage compared to the on-state (since the current level is lower) and for a higher supply voltage during the $I-V$ measurement (higher overdrive to overcome the degradation).

To avoid effects of (degradation of) the pass gates on our results, we chose to study the evolution of the threshold voltage $V_{\text {th }}$ instead of the on-current during the anneal.

\section{Measurement procedure}

Fig. 5 illustrates the measurement procedure used in this work. First, a time- $0 I-V$ of the DUT is measured. Then, HC 

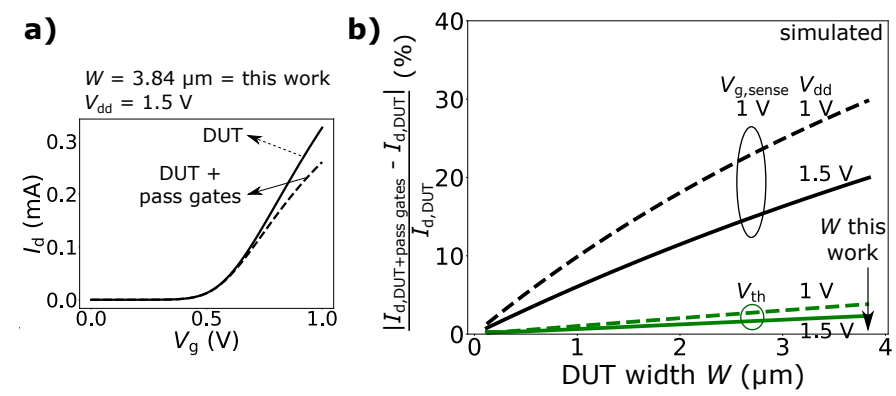

Fig. 3. a) Simulated linear $I-V$ of a DUT as available on the chip $\left(L_{\mathrm{g}}=\right.$ $40 \mathrm{~nm}, W=32 \times 120 \mathrm{~nm}$ ) without and with the source and drain pass gate included. The pass gates decrease the on-current. b) Simulated relative current decrease due to the pass gates for different (hypothetical) DUT widths

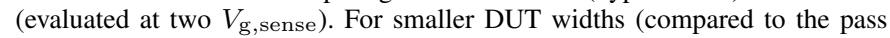
gate size), the pass gates affect the current of the DUTs less.
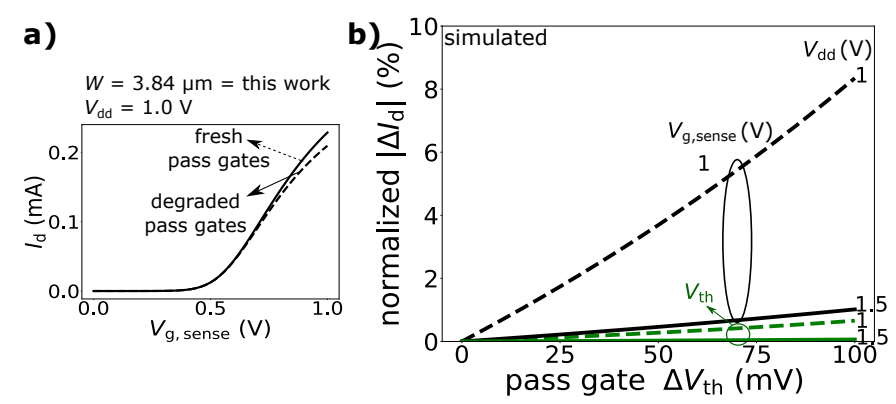

Fig. 4. a) Simulated linear $I-V$ of a DUT as available on the chip ( $L_{\mathrm{g}}=40$ $\mathrm{nm}, W=32 \times 120 \mathrm{~nm}$ ) without and with degradation of the pass gates. The pass gate degradation is modeled as a threshold voltage shift on the four pass gate transistors $\left(\Delta V_{\text {th,pass gate }}=100 \mathrm{mV}\right)$. b) Simulated relative current decrease due to degradation of the pass gates as in a) for different magnitudes of the pass gate degradation (evaluated at two $V_{\mathrm{g} \text {,sense }}$ ).

stress is applied at room temperature for $t_{\text {stress }}=10 \mathrm{ks}$. For the stress, the $V_{\mathrm{g}}<V_{\mathrm{d}}$ regime was chosen to create mainly interface states and avoid oxide charge trapping. The exact gate and drain voltages which were used vary from $0.6 \mathrm{~V}$ to $1 \mathrm{~V}$ and from $2 \mathrm{~V}$ to $2.25 \mathrm{~V}$ respectively and are indicated further on for every measurement separately. During stress, $I-V \mathrm{~s}$ are measured periodically (sense phase). We focus on the two shortest gate lengths $\left(L_{\mathrm{g}}=40,100 \mathrm{~nm}\right)$, because they result in the strongest $\mathrm{HCD}$.

Next, the anneal is started: a voltage is applied to the poly-Si heater and to the gate of the DUT. The total anneal time is $t_{\text {anneal }}=10 \mathrm{ks}$ and the total anneal temperatures $\left(T_{\text {ambient }}+\Delta T_{\text {poly-Si heater }}\right)$ are $140{ }^{\circ} \mathrm{C}, 180{ }^{\circ} \mathrm{C}, 230{ }^{\circ} \mathrm{C}$ and $270{ }^{\circ} \mathrm{C}$. During anneal, the poly-Si heaters are switched off periodically to measure $I-V \mathrm{~s}$ at room temperature in order to sense the recovery. Annealed devices are not reused for a subsequent stress-anneal measurement, i.e. the combined stress and anneal sequence is always carried out on a fresh device (Fig. 5).

We report both unbiased (zero gate voltage) and biased (non-zero gate voltage) anneal measurements. For the unbiased case, we apply $V_{\mathrm{g} \text {,anneal }}=V_{\mathrm{s}, \text { anneal }}=V_{\mathrm{d} \text {,anneal }}=V_{\mathrm{b}, \text { anneal }}=$
$0 \mathrm{~V}$. For the biased case, the situation is different because of the pFET selector on the gate of the DUT (Fig. 2a). The gate voltage of this pFET selector is limited to $V \gtrsim-0.5 \mathrm{~V}$ in order not to apply too much forward bias to the bulk-source junction of the nFETs in some of the peripheral circuits. This makes applying a negative gate bias (below $V_{\mathrm{ss}}=0 \mathrm{~V}$ ) to the DUT difficult. Therefore, we apply a constant offset voltage $V_{\text {offset }}=0.7 \mathrm{~V}$ to the source and drain during the biased anneal to pass a negative $V_{\mathrm{gs} \text {,anneal }}=V_{\mathrm{g} \text {,anneal }}-V_{\text {offset }}$ with a positive $V_{\mathrm{g} \text {,anneal. }}$. The applied gate voltages during the biased anneal are $V_{\mathrm{g} \text {,anneal }} \in\{0,0.4,0.7,1\} \mathrm{V}$ and so $V_{\mathrm{gs} \text {,anneal }}$ $\in\{-0.7,-0.3,0,0.3\} \mathrm{V}$. Note that the application of $V_{\mathrm{s} \text {,anneal }}$ $=V_{\mathrm{d} \text {,anneal }}=V_{\text {offset }}$ leads to a body bias, since the bulk of the DUT is grounded.

All $I$ - $V \mathrm{~s}$ are measured in linear mode $\left(V_{\mathrm{d}}=0.05 \mathrm{~V}\right)$. The threshold voltage is calculated using the max $-g_{\mathrm{m}}$ method at time- 0 and the constant-current method afterwards. Changes in $V_{\text {th }}$ during both stress and anneal are referenced to the time- 0 value.

\section{RESUlTS AND Discussion}

We first discuss HCD anneal at zero gate bias and then continue with the anneal at non-zero gate bias.

\section{A. Anneal at zero gate bias}

Fig. 6 shows the shift of the threshold voltage during $\mathrm{HC}$ stress. For the same stress condition, we observe a higher degradation for the $40 \mathrm{~nm}$ compared to the $100 \mathrm{~nm}$ devices, because of the higher lateral electric field in the former FET. Fig. 7 shows the subsequent anneal of the devices stressed in Fig. $6\left(V_{\mathrm{g} \text {,anneal }}=V_{\mathrm{s} \text {,anneal }}=V_{\mathrm{d} \text {, anneal }}=0 \mathrm{~V}\right)$. With higher anneal temperature, a higher portion of the threshold voltage shift at the end of stress $\left(\max \left(\Delta V_{\mathrm{th}}\right)\right)$ recovers. Since we use relatively low gate voltages for HCD stress $\left(V_{\mathrm{g} \text {,stress }} \leq 1 \mathrm{~V}\right.$ $\left.=V_{\mathrm{dd}, \text { nominal }}\right)$ and our devices have no high-k dielectric, the contribution of pBTI to $\Delta V_{\mathrm{th}}$ should be small and the HCD should be mainly caused by interface defects $\left(P_{\mathrm{b}}\right.$-centers). As a reference, we also show in Fig. 7 the post-stress time evolution of the threshold voltage shift for a device which did not undergo HCD anneal $\left(\Delta T_{\text {poly-Si heater }}=0{ }^{\circ} \mathrm{C}\right)$. We see that in this case there is only a very small recovery, meaning that the degradation is permanent at room temperature. This further points towards degradation consisting of interface defects.

Process-induced $P_{\mathrm{b}}$-centers have been intensively studied at the fundamental level. Using electron paramagnetic resonance, Brower [25] detected that $P_{\mathrm{b}}$-centers on (111) $\mathrm{Si}_{-} \mathrm{SiO}_{2}$ can be passivated in $\mathrm{H}_{2}$ and that the passivation follows a first order kinetics model (concentration of $P_{\mathrm{b}}$-centers decreases exponentially in time) with passivation energy $E_{\mathrm{a}}=1.66 \mathrm{eV}$. Stesmans [14] showed that this model is also applicable to $P_{\mathrm{b}}$-centers on the (100) $\mathrm{Si}-\mathrm{SiO}_{2}$ interface, provided that a Gaussian distribution with standard deviation $\sigma\left(E_{\mathrm{a}}\right)$ is taken into account for the passivation energy. This distribution originates from structural disorder in the $P_{\mathrm{b}}$-defects at the 


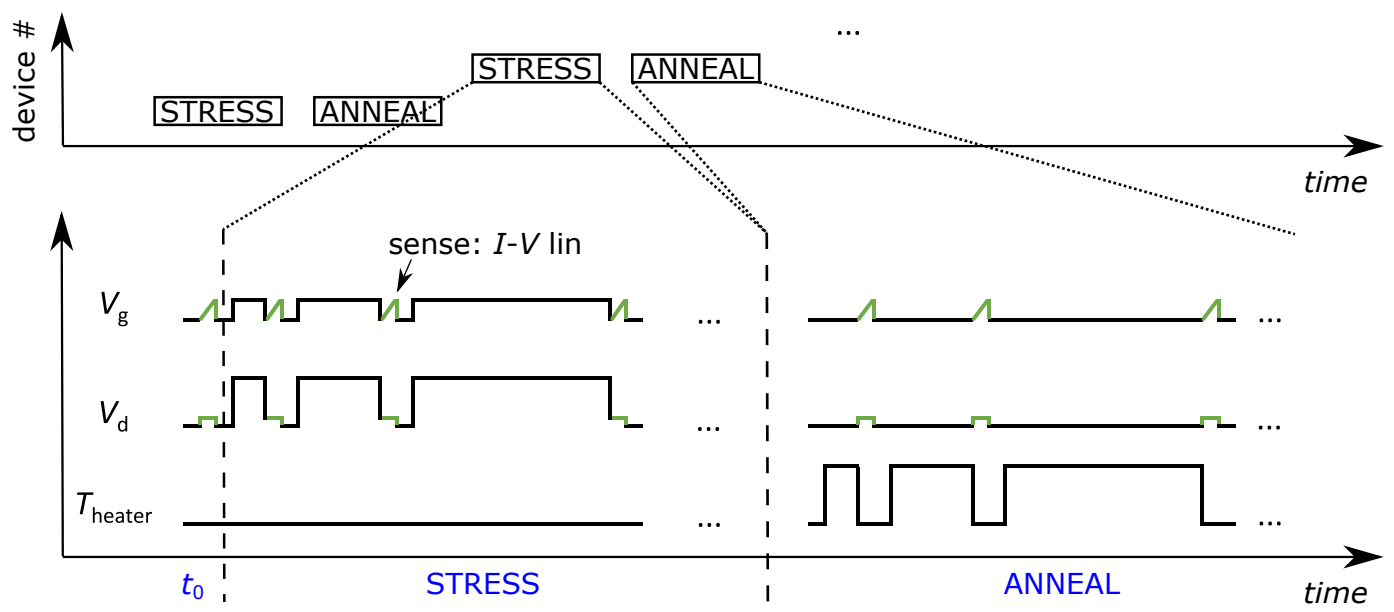

Fig. 5. Measurement scheme used in this work. For each device, the anneal phase is carried out after the stress phase. The indicated voltages in the anneal phase are for the unbiased anneal.

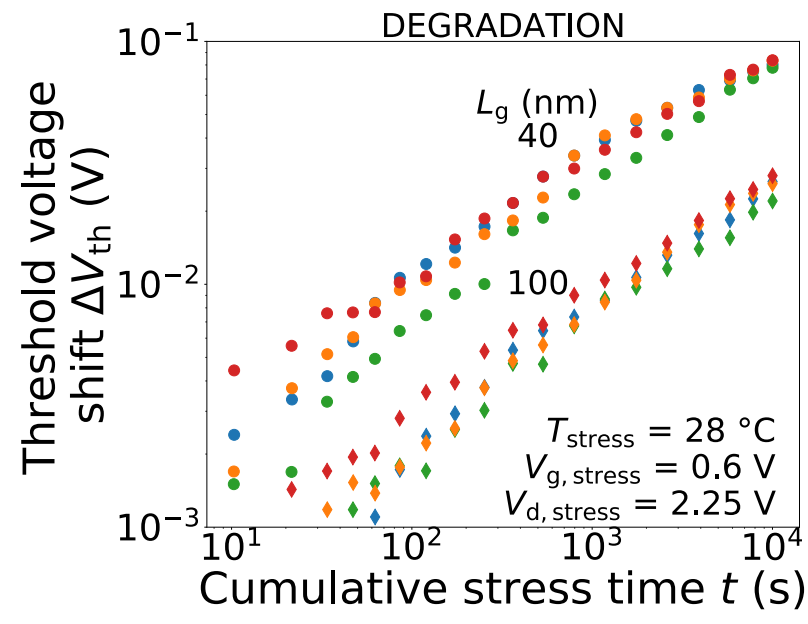

Fig. 6. Threshold voltage shift $\Delta V_{\text {th }}$ versus time due to HC stress for two gate lengths.

interface. In Stesmans' model, the concentration of $P_{\mathrm{b}}$-defects $\left[P_{\mathrm{b}}\right]$ evolves as function of anneal time $t_{\mathrm{H}}$ as:

$$
\begin{aligned}
& \frac{\left[P_{\mathrm{b}}\right]}{N_{0}}=\frac{1}{\sqrt{2 \pi} \sigma\left(E_{\mathrm{a}}\right)} \int_{-\infty}^{\infty} \exp \left(-\frac{\left(E-E_{\mathrm{a}}\right)^{2}}{2 \sigma\left(E_{\mathrm{a}}\right)^{2}}\right) \\
& \quad \times \exp \left(-k_{\mathrm{f}, 0}\left[\mathrm{H}_{2}\right] t_{\mathrm{H}} \exp \left(-\frac{E}{k T}\right)\right) \mathrm{d} E .
\end{aligned}
$$

Here, $N_{0}$ is the maximum density of $P_{\mathrm{b}}$-centers (i.e. at $t_{\mathrm{H}}=$ $0 \mathrm{~s}), k_{\mathrm{f}, 0}$ the prefactor of the forward rate constant, $\left[\mathrm{H}_{2}\right]$ the interfacial volume concentration of $\mathrm{H}_{2}$ and $k$ the Boltzmann's constant. For completeness, we mention that passivated $P_{\mathrm{b}}$ defects $\left(P_{\mathrm{b}} \mathrm{H}\right)$ dissociate again in a vacuum anneal with a dissociation energy $E_{\mathrm{a}}=2.56 \mathrm{eV}$ (value obtained for (111) $\left.\mathrm{Si}^{-\mathrm{SiO}_{2}}[26]\right)$.

The data in Fig. 7 can be fitted using (1), similar to Pobegen et al. [13] and de Jong et al. [6]. To this end, we approximate $\left[P_{\mathrm{b}}\right] \sim \Delta V_{\text {th }}$ and $N_{0} \sim \max \left(\Delta V_{\text {th }}\right)$. We did not include the reference curve at $T=28{ }^{\circ} \mathrm{C}$ in the fit, but note that the

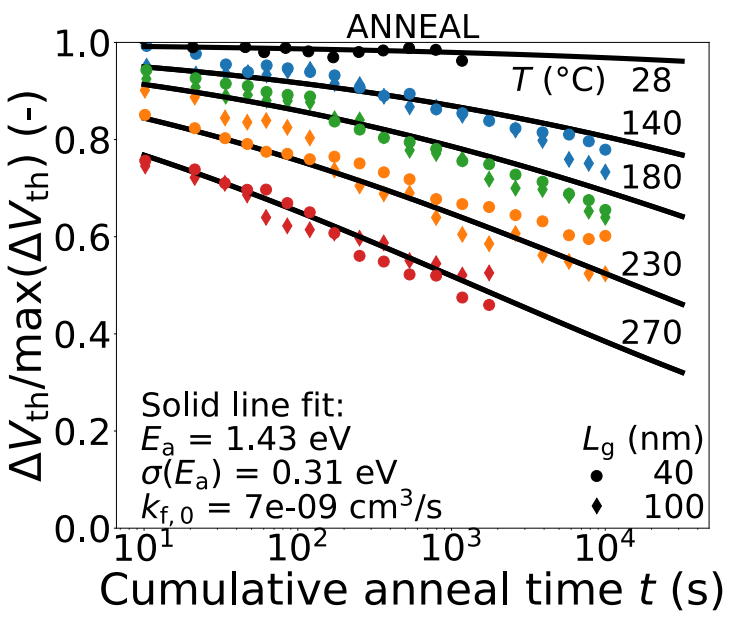

Fig. 7. Recovery of the $\mathrm{HC}$ induced threshold voltage shift $\Delta V_{\text {th }}$ from Fig. 6 versus time during anneal at five temperatures. The devices are biased at $V_{\mathrm{s} \text {, anneal }}=V_{\mathrm{d} \text {, anneal }}=V_{\mathrm{g} \text {, anneal }}=0 \mathrm{~V}$ and the indicated temperature is the total temperature $\left(T_{\text {ambient }}+\Delta T_{\text {poly-Si heater }}\right)$. The black lines are a fit (using the same parameter set) of the anneal data to (1). The curve for $T=28{ }^{\circ} \mathrm{C}$ corresponds to $\Delta T_{\text {poly-Si heater }}=0{ }^{\circ} \mathrm{C}$ (no $\mathrm{HCD}$ anneal) and is included as reference. This one device was stressed at a slightly lower $V_{\mathrm{d} \text {,stress }}=2.1 \mathrm{~V}\left(V_{\mathrm{g}, \text { stress }}=0.6 \mathrm{~V}\right)$.

observed slight recovery at room temperature is predicted by Stesmans' model. Table I summarizes our obtained values for the model parameters and compares them with the values of other groups. Our values for the activation energy $E_{\mathrm{a}}$ and its standard deviation $\sigma\left(E_{\mathrm{a}}\right)$ are in line with the previous works [6], [13], [14]. The value for the prefactor $k_{\mathrm{f}, 0}$ of the rate constant is smaller. We attribute this to possible inaccuracies in the used value for the interfacial volume concentration $\left[\mathrm{H}_{2}\right]$ $=10^{18} \mathrm{~cm}^{3} / \mathrm{s}$ of $\mathrm{H}_{2}$. As visible in Table I, there is also a large variation in the value of $k_{\mathrm{f}, 0}$ between different studies. We speculate that the $\mathrm{H}_{2}$ involved in the HCD anneal is residual $\mathrm{H}_{2}$ trapped in the gate stack during the passivation steps of the chip fabrication. 
TABLE I

OVERVIEW OF THE ORIGINAL VALUES FOR THE MODEL PARAMETERS IN THE PASSIVATION MODEL OF STESMANS (AND BROWER) FOR PROCESS-INDUCED $P_{\mathrm{b}}$-CENTERS AND THE VALUES OBTAINED IN THIS WORK AND BY OTHER GROUPS WHEN APPLYING THE MODEL TO HCD ANNEAL.

\begin{tabular}{|c|c|c|l|l|l|}
\hline Ref. & $P_{\mathrm{b}}$ origin & Study & $\begin{array}{l}E_{\mathrm{a}} \\
(\mathrm{eV})\end{array}$ & $\begin{array}{l}\sigma\left(E_{\mathrm{a}}\right) \\
(\mathrm{eV})\end{array}$ & $\begin{array}{l}k_{\mathrm{f}, 0} \\
\left(10^{-6} \mathrm{~cm}^{3} / \mathrm{s}\right)\end{array}$ \\
\hline$[25]$ & Oxidation & EPR $\left(P_{\mathrm{b}}\right)$ & $1.66 \pm 0.06$ & $/$ & $1.94_{-1}^{+2}$ \\
{$[14]$} & Oxidation + & ESR $\left(P_{\mathrm{b} 0}\right)$ & $1.51 \pm 0.04$ & $0.14 \pm 0.02$ & $1.43_{-0.6}^{+0.6}$ \\
& vac. anneal & ESR $\left(P_{\mathrm{b} 1}\right)$ & $1.57 \pm 0.04$ & $0.15 \pm 0.03$ & \\
\hline$[13]$ & $\mathrm{HCD}$ & $\mathrm{CP}$ & $1.6 \pm 0.1$ & $0.20 \pm 0.02$ & $7_{-6.4}^{+83} \times 10^{2}$ \\
{$[6]$} & $\mathrm{HCD}$ & $I-V \mathrm{~s}$ & 1.39 & 0.369 & $2 \times 10^{-1}$ \\
\hline $\begin{array}{c}\text { This } \\
\text { work }\end{array}$ & $\mathrm{HCD}$ & $I-V \mathrm{~s}$ & 1.43 & 0.31 & $7 \times 10^{-3}$ \\
\hline
\end{tabular}

\section{B. Anneal at non-zero gate bias}

Next, we investigate the influence on the HCD recovery of a gate bias applied to the DUT during the anneal (Fig. 8). We observe a larger relative recovery of the threshold voltage shift for more negative $V_{\mathrm{gs} \text {,anneal. }}$. This trend is seen for the three temperatures studied $\left(T=140,180,230{ }^{\circ} \mathrm{C}\right)$. To make the influence of the gate bias better visible, we extracted the $V_{\mathrm{gs}, \text { anneal }}$ dependence of the passivation energy. To this end, we fitted for each $V_{\mathrm{gs} \text {,anneal }}$ separately the data in Fig. 8 to Stesmans' model, using the values from Fig. 7 for $\sigma\left(E_{\mathrm{a}}\right)$ and $k_{\mathrm{f}, 0}$. Fig. 9 shows that the passivation energy decreases with decreasing $V_{\mathrm{gs}, \text { anneal }}$.

The observed dependence on $V_{\mathrm{gs} \text {,anneal }}$ is consistent with the trend reported and explained by Ragnarsson et al. [15] for process-induced $P_{\mathrm{b}}$-defects. Ragnarsson et al. found that the passivation of $P_{\mathrm{b}}$-centers depends on their charge state and therefore implicitly on the position of the Fermi-level and the gate bias. According to theoretical work by Edwards [27] (for the (111) $\mathrm{Si} / \mathrm{SiO}_{2}$-interface), the passivation rate of positively charged $P_{\mathrm{b}}$-defects is higher than for neutral ones, which in turn is higher than for negatively charged ones. So, decreasing the gate bias moves the Fermi-level at the interface closer to the valence band edge, depopulating the upper energy level (becoming more neutral) and the lower energy level (becoming more positively charged) of the $P_{\mathrm{b}}$-defects (see band diagrams in Fig. 9). These two charge transitions increase the passivation rate and the anneal.

We note that the value for $E_{\mathrm{a}}$ at $V_{\mathrm{gs} \text {, anneal }}=0 \mathrm{~V}$ obtained in Fig. 9 is slightly smaller than the one obtained in Fig. 7, which also corresponds to zero bias anneal. We attribute the difference to the fact that these two zero bias cases are not exactly identical. In Fig. 7 , we have $V_{\mathrm{s} \text {,anneal }}=V_{\text {d,anneal }}=$ $V_{\mathrm{g} \text {,anneal }}=V_{\mathrm{b} \text {,anneal }}=0 \mathrm{~V}$, while in Fig. 9 , we have $V_{\mathrm{s} \text {,anneal }}$ $=V_{\mathrm{d} \text {,anneal }}=V_{\mathrm{g}, \text { anneal }}=0.7 \mathrm{~V} \neq V_{\mathrm{b} \text {,anneal }}=0 \mathrm{~V}$. Hence, there is no body bias in the measurement of Fig. 7, while there is reverse body bias in the measurement of Fig. 9. The reverse body bias increases the threshold voltage compared to no body bias. TCAD simulations (not shown) indicate that at $V_{\mathrm{gs}, \text { anneal }}=0 \mathrm{~V}$ the Fermi-level is closer to the valence band edge in the case of reverse body bias compared to zero body bias. So, the energy levels of the $P_{\mathrm{b}}$-defects should be more depopulated at $V_{\mathrm{gs}, \text { anneal }}=0 \mathrm{~V}$ for reverse body bias (Fig. 9), leading to a smaller passivation energy compared to zero body bias (Fig. 7).

For the reliability modeling of MOSFETs, more specifically interface state modeling in $\mathrm{HCD}$, the $\mathrm{Si}-\mathrm{H}$ bond breakage model (schematically depicted in Fig. 10) is generally used [18], [19]. In this model, Si-H bonds can be broken in two ways (thin arrow in Fig. 10). The first option is that a highly energetic carrier (i.e. with energy corresponding to at least the $\mathrm{Si}-\mathrm{H}$ bond dissociation energy $E_{\mathrm{a}}$ ) collides with the bond and breaks it in one strike. Alternatively, multiple less energetic carriers (i.e. with energy corresponding to the vibrational mode energy $\hbar \omega$ ) can induce vibrational excitations of the $\mathrm{Si}-\mathrm{H}$ bond upon collision, increasing its energy until it eventually breaks. Since HCD can be recovered at elevated temperature, the passivation process of broken $\mathrm{Si}-\mathrm{H}$ bonds needs to be included in this model (thick orange arrow in Fig. 10). The measurements in this work indicate that the passivation energy to be used in this model is gate bias dependent.

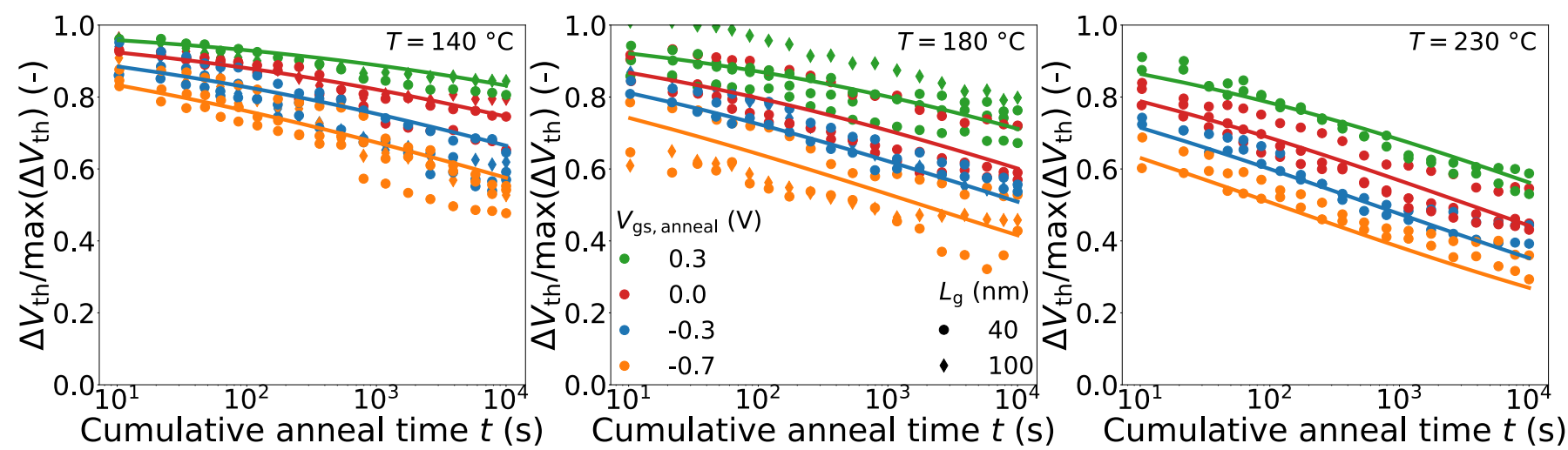

Fig. 8. Influence of gate bias $V_{\mathrm{gs}}$,anneal on the relative recovery of the threshold voltage shift caused by HC stress at three temperatures. The lines are calculated using the passivation model of Stesmans, with $\sigma\left(E_{\mathrm{a}}\right)$ and $k_{\mathrm{f}, 0}$ equal to the value in Fig. 7 and $E_{\mathrm{a}}$ fitted for each $V_{\mathrm{gs}}$, anneal separately (see Fig. 9). Stress conditions are $V_{\mathrm{g} \text {,stress }}=0.6 \mathrm{~V}$ and $V_{\mathrm{d} \text {,stress }}=2.1 \mathrm{~V}$ for the $100 \mathrm{~nm}$ FETs and $V_{\mathrm{g}, \text { stress }}=1 \mathrm{~V}$ and $V_{\mathrm{d} \text {,stress }}=2 \mathrm{~V}$ for the $40 \mathrm{~nm}$ FETs. Stress was done at room temperature for a total time of $10 \mathrm{ks}$ for each device. 


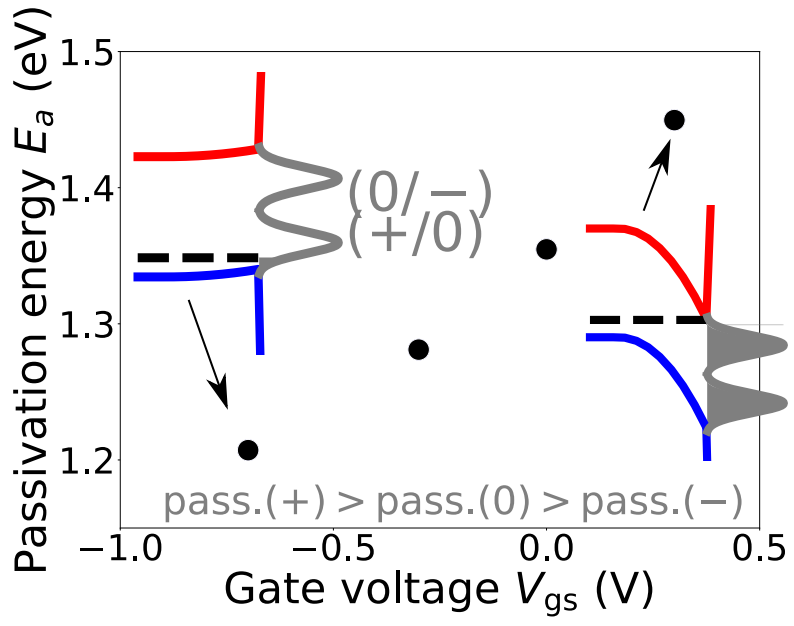

Fig. 9. Passivation energy $E_{\mathrm{a}}$ versus gate bias $V_{\mathrm{gs} \text {,anneal }}$ calculated by fitting Stesmans' model to the data in Fig. 8. The $P_{\mathrm{b}}$ passivation depends on the

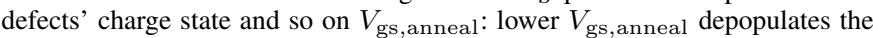
$P_{\mathrm{b}}$ energy levels, increasing their passivation [15] (see band diagrams, not representing the actual device).

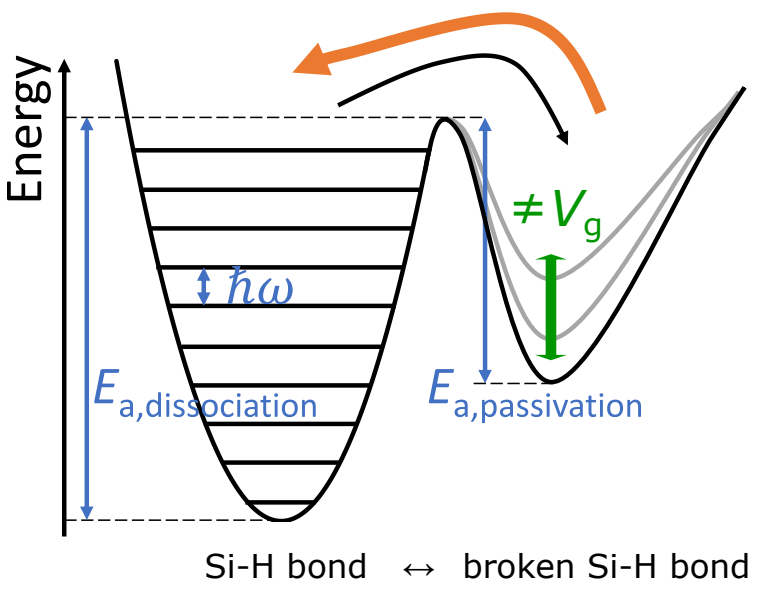

Fig. 10. Oscillator potential for Si-H bond breakage, used for modeling HCD [18], [19]. HCD anneal is attributed to a repassivation (thick arrow) of the formed broken $\mathrm{Si}-\mathrm{H}$ bonds $\left(P_{\mathrm{b}}\right.$-centers). The measurements in this work show that a gate bias dependent passivation energy needs to be considered in the $\mathrm{Si}-\mathrm{H}$ bond breakage model.

\section{CONCLUSions}

We performed HCD anneal measurements on a commercial $40 \mathrm{~nm}$ bulk CMOS technology using poly-Si heaters. Consistent with previous findings, we find that the anneal is described using Stesmans' model for passivation of $P_{\mathrm{b}}$-centers in $\mathrm{H}_{2}$. The HCD anneal improves with negative gate bias, consistent with studies on biased passivation of process-induced $P_{\mathrm{b}}$ defects. We extracted the bias dependence of the passivation energy of the $P_{\mathrm{b}}$-centers in Stesmans' model and find it to decrease with decreasing gate voltage.

\section{ACKNOWLEDGMENTS}

Michiel Vandemaele is supported by a PhD Fellowship of the Research Foundation - Flanders (Belgium) (application number $11 \mathrm{~A} 3619 \mathrm{~N})$. Stanislav Tyaginov is supported by the
European Union's Horizon 2020 research and innovation programme (Marie Sklodowska-Curie grant agreement number 794950).

\section{REFERENCES}

[1] S. Novak et al., "Transistor aging and reliability in $14 \mathrm{~nm}$ tri-gate technology," in 2015 IEEE International Reliability Physics Symposium (IRPS), pp. 2F-2, IEEE, 2015.

[2] A. Rahman, J. Dacuna, P. Nayak, G. Leatherman, and S. Ramey, "Reliability studies of a $10 \mathrm{~nm}$ high-performance and low-power CMOS technology featuring $3^{\text {rd }}$ generation finFET and $5^{\text {th }}$ generation HK/MG," in 2018 IEEE International Reliability Physics Symposium (IRPS), pp. 6F-4, IEEE, 2018.

[3] R. Annunziata, G. Dalla Libera, E. Ghio, and A. Maggis, "Annealing of hot carrier damaged double metal MOSFET," in 1989 IEEE European Solid State Device Research Conference (ESSDERC), pp. 715-718, IEEE, 1989.

[4] C. H. Ling, L. K. Ah, W. K. Choi, S. E. Tan, and D. S. Ang, "Hotelectron degradation in nMOSFETs: results from temperature anneal," IEEE Transactions on Electron Devices, vol. 41, no. 7, pp. 1303-1305, 1994.

[5] M. J. de Jong, C. Salm, and J. Schmitz, "Observations on the recovery of hot carrier degradation of hydrogen/deuterium passivated nMOSFETs," Microelectronics reliability, vol. 76, pp. 136-140, 2017.

[6] M. J. de Jong, C. Salm, and J. Schmitz, "Towards understanding recovery of hot-carrier induced degradation," Microelectronics reliability, vol. 88, pp. 147-151, 2018.

[7] M. J. de Jong, C. Salm, and J. Schmitz, "Recovery after hot-carrier injection: slow versus fast traps," Microelectronics Reliability, p. 113318, 2019.

[8] J.-W. Han, M. Kebaili, and M. Meyyappan, "System on microheater for on-chip annealing of defects generated by hot-carrier injection, bias temperature instability, and ionizing radiation," IEEE Electron Device Letters, vol. 37, no. 12, pp. 1543-1546, 2016.

[9] J.-W. Han, R. Peterson, D.-I. Moon, D. G. Senesky, and M. Meyyappan, "Monolithically integrated microheater for on-chip annealing of oxide defects," IEEE Electron Device Letters, vol. 38, no. 7, pp. 831-834, 2017.

[10] G.-B. Lee et al., "A novel technique for curing hot-carrier-induced damage by utilizing the forward current of the PN-junction in a MOSFET," IEEE Electron Device Letters, vol. 38, no. 8, pp. 1012-1014, 2017.

[11] J.-Y. Park, J. Hur, and Y.-K. Choi, "Demonstration of a curable nanowire finFET using punchthrough current to repair hot-carrier damage," IEEE Electron Device Letters, vol. 39, no. 2, pp. 180-183, 2018.

[12] J.-Y. Park et al., "Self-curable gate-all-around MOSFETs using electrical annealing to repair degradation induced from hot-carrier injection," IEEE Transactions on Electron Devices, vol. 63, no. 3, pp. 910-915, 2016.

[13] G. Pobegen, S. Tyaginov, M. Nelhiebel, and T. Grasser, "Observation of normally distributed energies for interface trap recovery after hot-carrier degradation," IEEE Electron Device Letters, vol. 34, no. 8, pp. 939-941, 2013.

[14] A. Stesmans, "Passivation of $P_{\mathrm{b} 0}$ and $P_{\mathrm{b} 1}$ interface defects in thermal (100) $\mathrm{Si} / \mathrm{SiO}_{2}$ with molecular hydrogen," Applied Physics Letters, vol. 68 , no. 15 , pp. 2076-2078, 1996.

[15] L.-Å. Ragnarsson and P. Lundgren, "Electrical characterization of $P_{\mathrm{b}}$ centers in (100) $\mathrm{Si}-\mathrm{SiO}_{2}$ structures: the influence of surface potential on passivation during post metallization anneal," Journal of Applied Physics, vol. 88, no. 2, pp. 938-942, 2000.

[16] N. D. Young, "The formation and annealing of hot-carrier-induced degradation in poly-Si TFTs, MOSFETs, and SOI devices, and similarities to state-creation in $\alpha \mathrm{Si}: \mathrm{H}$," IEEE Transactions on Electron Devices, vol. 43, no. 3, pp. 450-456, 1996.

[17] C. R. Parthasarathy et al., "New insights into recovery characteristics during pMOS NBTI and CHC degradation," IEEE Transactions on Device and Materials Reliability, vol. 7, no. 1, pp. 130-137, 2007.

[18] C. Guerin, V. Huard, and A. Bravaix, "General framework about defect creation at the $\mathrm{Si} / \mathrm{SiO}_{2}$ interface," Journal of Applied Physics, vol. 105, no. 11 , p. $114513,2009$.

[19] S. Tyaginov et al., "Physical modeling of hot-carrier degradation for short-and long-channel MOSFETs," in 2014 IEEE International Reliability Physics Symposium, pp. XT-16, IEEE, 2014. 
[20] D.-I. Moon et al., "Sustainable electronics for nano-spacecraft in deep space missions," in 2016 IEEE International Electron Devices Meeting (IEDM), pp. 31-8, IEEE, 2016.

[21] X. Wang et al., "Silicon odometers: compact in situ aging sensors for robust system design," IEEE Micro, vol. 34, no. 6, pp. 74-85, 2014.

[22] K.-H. Chuang et al., "A multi-bit/cell PUF using analog breakdown positions in CMOS," in 2018 IEEE International Reliability Physics Symposium (IRPS), pp. P-CR, IEEE, 2018.

[23] J. Franco, B. Kaczer, and G. Groeseneken, "Poly-Si heaters for ultra-fast local temperature control of on-wafer test structures," Microelectronic Engineering, vol. 114, pp. 47-51, 2014.

[24] http://www.cadence.com/en_US/home/tools/custom-ic-analog-rfdesign/circuit-simulation/spectre-simulation-platform.html.

[25] K. L. Brower, "Kinetics of $\mathrm{H}_{2}$ passivation of $P_{\mathrm{b}}$ centers at the (111) $\mathrm{Si}-\mathrm{SiO}_{2}$ interface," Physical Review B, vol. 38, no. 14, p. 9657, 1988.

[26] K. L. Brower, "Dissociation kinetics of hydrogen-passivated (111) Si$\mathrm{SiO}_{2}$ interface defects," Physical Review B, vol. 42, no. 6, p. 3444, 1990.

[27] A. H. Edwards, "Interaction of $\mathrm{H}$ and $\mathrm{H}_{2}$ with the silicon dangling orbital at the $\langle 111\rangle \mathrm{Si} / \mathrm{SiO}_{2}$ interface," Physical Review B, vol. 44, no. 4, p. $1832,1991$. 